\title{
Concomitant cervical and transperineal parametrial high-dose-rate brachytherapy boost for locally advanced cervical cancer
}

\author{
Caroline Bailleux, MD!, Alexander Tuan Falk, MD!, Marie-Eve Chand-Fouche, MD!, Mathieu Gautier, MSc!, \\ Emmanuel Barranger, MD, PhD², Jean-Michel Hannoun-Levi, MD, PhD' \\ 'Department of Radiation Oncology, ${ }^{2}$ Department of Surgery, Antoine Lacassagne Cancer Center, University of Nice-Sophia, Nice, France
}

\begin{abstract}
Purpose: There is no consensus for parametrial boost technic while both transvaginal and transperineal approaches are discussed. A prototype was developed consisting of a perineal template, allowing transperineal needle insertion. This study analyzed acute toxicity of concomitant cervical and transperineal parametrial high-dose-rate brachytherapy (HDRB) boost for locally advanced cervical cancer.

Material and methods: From 01.2011 to 12.2014, 33 patients (pts) presenting a locally advanced cervical cancer with parametrial invasion were treated. After the first course of external beam radiation therapy with cisplatinum, HDRB was performed combining endocavitary and interstitial technique for cervical and parametrial disease. Post-operative delineation (CTV, bladder, rectum, sigmoid) and planification were based on CT-scan/MRI. HDRB was delivered in 3-5 fractions over 2-3 consecutive days. Acute toxicities occurring within 6 months after HDRB were retrospectively reviewed.

Results: Median age was 56.4 years (27-79). Clinical stages were: T2b $=23$ pts $(69.7 \%), \mathrm{T} 3 \mathrm{a}=1 \mathrm{pt}(3 \%), \mathrm{T} 3 \mathrm{~b}=6 \mathrm{pts}$ $(18.2 \%)$, and $\mathrm{T} 4 \mathrm{a}=3 \mathrm{pts}(9.1 \%)$. Median HDRB prescribed dose was 21 Gy (21-27). Median $\mathrm{CTV}_{\mathrm{CT}}(16 \mathrm{pts})$ and $\mathrm{HR}_{-\mathrm{CTV}} \mathrm{MRI}(17 \mathrm{pts})$ were $52.6 \mathrm{cc}(28.5-74.3)$, $31.9 \mathrm{cc}(17.1-58)$, respectively. Median EQD2 ${ }_{\alpha \beta 10}$ for $\mathrm{D} 90_{\mathrm{CTV}}$ and D90 ${ }_{\text {HR-CTV }}$ were 82.9 Gy (78.2-96.5), 84.8 Gy (80.6-91.4), respectively. Median EQD2 ${ }_{\alpha \beta 3}(\mathrm{CT} / \mathrm{MRI})$ for $\mathrm{D}_{2 \mathrm{cc}}$ bladder, rectum and sigmoid were 75.5 Gy (66.6-90.9), 64.4 Gy (51.9-77.4), and 60.4 Gy (50.9-81.1), respectively. Median follow-up was 14 months (ranged 6-51). Among the 24 pts with MFU = 24 months, 2-year LRFS rate, RRFS, and OS were $86.8 \%, 88.8 \%$, and $94.1 \%$, respectively. The rates of acute genitourinary and gastrointestinal toxicities were 36\% (G1 dysuria $=8$ pts, $\mathrm{G} 2$ infection $=2 \mathrm{pts}, \mathrm{G} 3$ infection $=2 \mathrm{pts})$, and 27\% (G1 diarrhea $=9 \mathrm{pts})$, respectively. One patient presented vaginal bleeding at the time of applicator withdrawal (G3-blood transfusion); no bleeding was observed due to the parametrial implant.

Conclusions: Concomitant cervical and transperineal parametrial HDRB boost for locally advanced cervical cancer appears feasible and safe with no specific acute toxicity compare to cervical HDRB alone. Longer follow-up and larger patient cohort will be needed.
\end{abstract}

Key words: boost, brachytherapy, cervical cancer, parametrial invasion, radiotherapy.

\section{Purpose}

Cervical cancer is the fourth most common cancer in women in the world, with an estimated 528,000 new cases and 266,000 deaths in 2012 [1]. Surgery is the standard treatment for early stage cervical cancer $(\leq \mathrm{T} 1 \mathrm{~b} 1)$. For locally advanced tumors, gold-standard treatment consists in a weekly platin-based chemotherapy combined with external beam radiation therapy (EBRT) followed by brachytherapy (BT) boost [2].

Brachytherapy remains a key component for locally advanced stage cervical cancer. Indeed, Han et al. publish- ed the results of a US brachytherapy survey showing that the use of BT decreased significantly from $83 \%$ in 1988 to $58 \%$ in $2009(p<0.001)$ [3]. The authors have shown that during the same period, BT was independently associated with significantly higher disease-specific survival (HR 0.64, 95\% CI: 0.57-0.71) and overall survival (HR 0.66, 95\% CI: 0.60-0.74), and should be implemented in all feasible cases [3]. However, due to important technological improvements available with high-tech EBRT, some authors proposed to use new EBRT techniques in place of BT. Gill et al. reported that compared to BT boost using intensity
Address for correspondence: Jean-Michel Hannoun-Levi, MD, PhD, Department of Radiation Therapy, Antoine Lacassagne Cancer Center, 33 Avenue de Valombrose, 06189 Nice Cedex, France, University of Nice-Sophia, 28 Avenue Valrose, 06103 Nice Cedex, France, phone: +33 492031271 , fax: +33 492031 570, e-mail: jean-michel.hannoun-levi@nice.unicancer.fr
Received: 20.08.2015

Accepted: 06.01.2016

Published: 26.02.2016 
modulated radiation therapy or stereotactic body radiation therapy, resulted in significantly lower OS (HR 1.86, 95\% CI: 1.35-2.55, $p<0.01)$, confirming that BT boost was a critical component of locally advanced cervix carcinoma treatment [4].

Different applicators, such as ovoid or ring tandems, molds, and vaginal cylinders with intra-uterine tubes are commonly used. In most cases, a typical pear-shaped isodose results from the intra-vaginal and intra-uterine sources but in case of larger tumors and especially with parametrial involvement, dose distribution is often non-optimal. In order to improve parametrial coverage, some applicators combining intracavitary (IC) and interstitial (IS) approach were proposed without increasing the dose to the organs at risk (OAR): Vienna applicator: ring applicator plus 6 to 9 interstitial needles around intrauterine tandem (Nucletron, an Elekta company, Elekta AB, Stockholm, Sweden) [5,6], Utrecht applicator: tandem ovoids +10 needles (Nucletron, an Elekta company, Elekta AB, Stockholm, Sweden) [7], and Nice Gynecologic Applicator: NGA uterine tandem + vaginal cylinder +8 needles within the cervix (Nucletron, an Elekta company, Elekta AB, Stockholm, Sweden) [8].

Currently, there is no consensus for parametrial boost technic while both transvaginal and transperineal approaches are discussed. Recently, for locally advanced tumors with parametrial involvement, interstitial BT parametrial boost using trans-vaginal approach was shown to be dosimetrically superior to EBRT parametrial boost in terms of target volume coverage and OAR sparing [9]. In order to improve the parametrium coverage, we developed a prototype consisting of a dedicated perineal template fixed to the NGA allowing a transperineal needle insertion.

This study aimed to assess feasibility, reproducibility, and acute toxicity of concomitant cervical and transperineal parametrial high dose rate brachytherapy (HDRB) boost for locally advanced cervix carcinomas.

\section{Material and methods}

\section{Patient features}

From January 2011 to December 2014, 33 patients (pts) presenting a histology proven locally advanced cervix carcinoma with parametrial invasion were retrospectively analyzed regarding dosimetric data, acute toxicities, and early clinical outcomes. All patients underwent parametrial HDRB boost. A local ethics committee initially approved this therapeutic approach.

All patients had a clinical exam and follow-up performed by trained physicians. Patients underwent cervical, vaginal, and rectal examination. Computed tomography scan (CT), pelvic magnetic resonance imaging (MRI), and positron emission tomography/computed tomography (PET) were performed. Para-aortic lymph node dissection was performed at the discretion of the physician in order to improve cancer staging and EBRT target volume definition. Tumors were staged using UICC-TNM classification [10].

\section{Treatment features}

After the first course of EBRT with concomitant platin-based chemotherapy, a single implantation of HDRB was performed combining endocavitary and interstitial technique for cervical disease, and transperineal interstitial approach for parametrial extension.

\section{Concomitant radio-chemotherapy}

The first part of the treatment consisted of EBRT with concurrent chemotherapy. EBRT delivered a total dose of 45 to 46 Gy (ICRU point) in 25 or 23 fractions, based on a 3-dimensional conformal technique with or without modulated intensity using $>10 \mathrm{MV}$ X-photons. Gross tumor volume (GTV) was determined clinically and radiologically for the primary tumor and any pathological nodes. The clinical target volume (CTV) included the GTV and surrounding subclinical disease. CTV involved the uterus, parametrial tissue, upper vagina (or whole vagina for T3a disease), and broad and utero-sacral ligaments. All pelvic-lymph nodal stations were included in the CTV with a recommended $7 \mathrm{~mm}$ margin around the blood vessels. Planning target volume (PTV) included the CTV plus margin to account for internal organ movements, setup, and delivery uncertainties. Concomitant chemotherapy mainly consisted in weekly platin-based chemotherapy using the following protocols: weekly cisplatin $40 \mathrm{mg} / \mathrm{m}^{2}$, weekly carboplatin $100 \mathrm{mg} / \mathrm{m}^{2}$, weekly cisplatin $40 \mathrm{mg} / \mathrm{m}^{2}+$ gemcitabin $125 \mathrm{mg} / \mathrm{m}^{2}$, monthly cisplatin $50-75 \mathrm{mg} / \mathrm{m}^{2}$ for 1 day + 5-fluorouracile $1000 \mathrm{mg} / \mathrm{m}^{2}$ from day 2 to day 5 , weeks 1 and 5 .

\section{Brachytherapy}

Brachytherapy was planned after the completion of radio-chemotherapy (RCT) with a time interval between the last RCT session and brachytherapy of less than 21 days. The single brachytherapy implantation was performed under general anesthesia starting by a gynecologic examination in order to evaluate the clinical response obtained after RCT.

\section{Endocavitary and interstitial procedure for central disease}

The endocavitary and interstitial procedure for central disease was already described [8]. Briefly, uterine tandem was introduced in the uterus cavity; it is then preceded to the placement of a vaginal cylinder including eight equidistant channels allowing the placement of eight plastic needles (240 mm Sharp Needles ${ }^{\mathrm{TM}}$; Elekta company, Elekta AB, Stockholm, Sweden) using flexible chucks, within the cervical tissue (40 mm depth). Vaginal cylinder was sutured to the skin through a skin suture template and a blocking needle template fixed the needles into the vaginal cylinder (Figure 1A).

\section{Interstitial procedure for parametrial disease}

The perineal template punched by a total of 6 holes was fixed to the vaginal cylinder before its introduction into the vaginal cavity. Due to the ischium, this perineal 

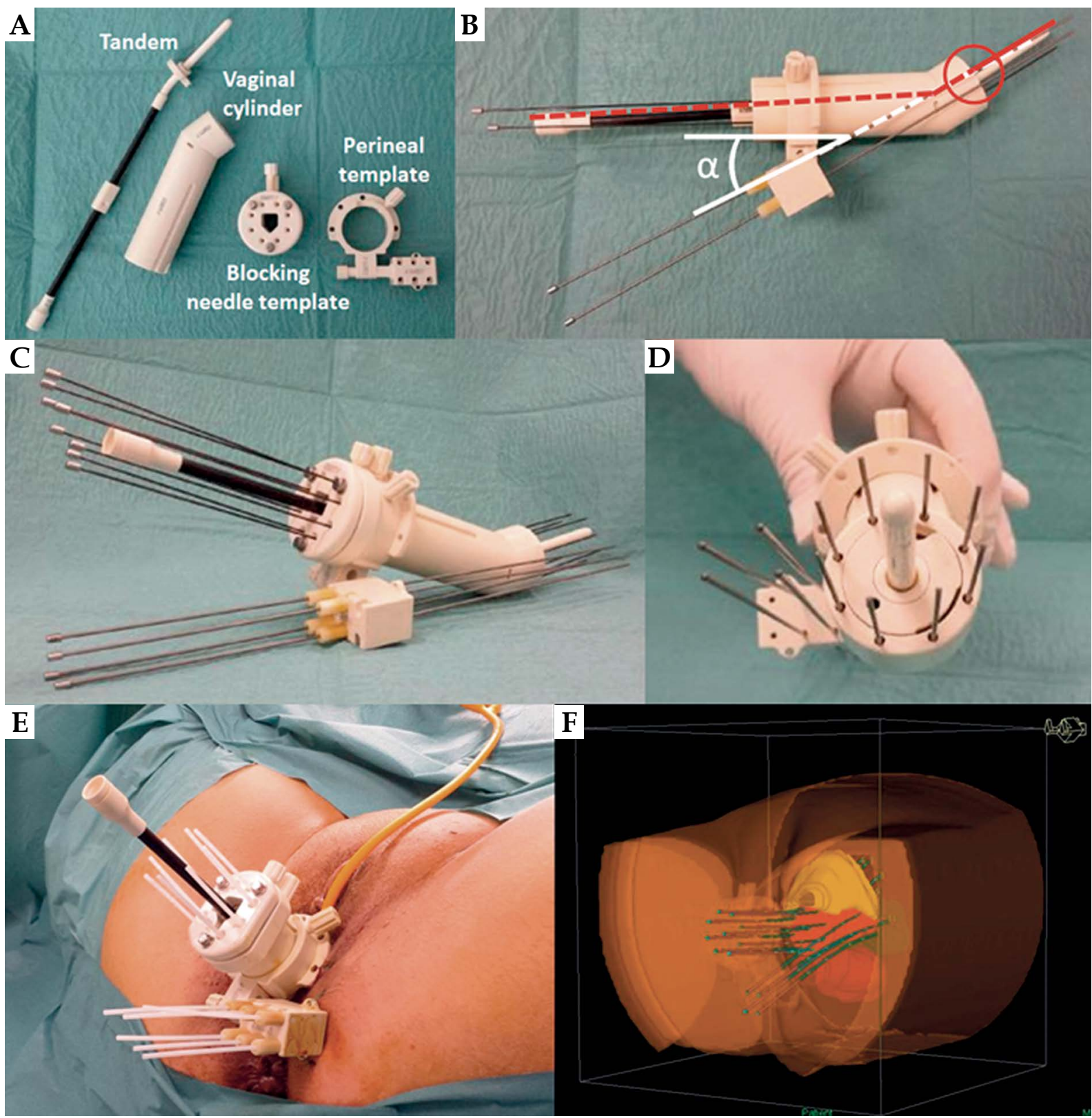

Fig. 1. Overview of the applicator allowing concomitant endocavitary and interstitial implant for cervical and parametrial disease. A) Different pieces of the applicator before assembling. B) Assembled applicator showing the oblique orientation ( $\alpha$ angle) of the perineal template allowing the upper-inner parametrial needle to cross the uterus tandem at the distal part of the vaginal cylinder. C) Overview of the assembled applicator. D) Frontal proximal view of the assembled applicator. E) Post-operative view of the applicator showing vaginal and parametrial needles. F) Post-operative 3D reconstruction

template has to be placed under the bone structure but has also to be obliquely oriented allowing the upper-inner parametrial needle to cross the uterus tandem at the distal part of the vaginal cylinder (Figures 1B, 1C, 1D). Once the perineal template was fixed on the skin perineum, parametrial transperineal $240 \mathrm{~mm}$ needles (Sharp Needles $^{\mathrm{TM}}$; Elekta Company, Elekta AB, Stockholm, Sweden) were placed into the parametrium through the perineal template, using rigid chucks. Number of needles (2 to 6) was defined according to the pre-treatment target volume and clinical examination performed during HDRB (Figure 1E).
After recovery, post-implant planning CT scan (until 12.2013), then MRI (after 01.2014) was performed for treatment planning purposes. CTV (CT scan planning), highrisk CTV (HR-CTV), and intermediate-risk (IR-CTV) (MRI planning) as well as OARs (bladder, rectum, and sigmoid) were delineated according to the Gyn GEC-ESTRO recommendations [11] (Figure 1F).

HDRB dose was delivered in respect to the current Gyn. GEC-ESTRO dose constraint rules recommending EQD2 ${ }_{\alpha \beta 10}$ for D90 $0_{\text {HR-CTV }}>80$ Gy and EQD2 ${ }_{\alpha \beta 3}$ for $\mathrm{D} 2 \mathrm{cc}_{\text {bladder }}<90 \mathrm{~Gy}$, and for D2 $\mathrm{cc}_{\text {rectum/sigmoid }}<75 \mathrm{~Gy}$. Brachytherapy dose was delivered in 3 to 5 fractions over 
2 to 3 consecutive days. Total dose and dose per fraction changed also according to the year of treatment. Between 2011 and 2012, the total delivered dose was 25 Gy in 5 fractions over 3 days, while 1 fraction of 7 Gy was delivered the day of implantation and 2 fractions of $4.5 \mathrm{~Gy}$ twice daily (at least 6 hours apart) for two days (EQD2 ${ }_{\alpha \beta 10}$ $\left.=32 \mathrm{~Gy} / \mathrm{EQD} 2_{\alpha \beta 3}=41 \mathrm{~Gy}\right)$. Since May 2013, in order to make brachytherapy less uncomfortable, the number of fractions was reduced from 5 to 3 with a total dose of 21 Gy in 3 fractions over 2 days with 1 fraction of 7 Gy delivered the day of implantation, and 2 fractions of $7 \mathrm{~Gy}$ (at least 6 hours apart) the day after $\left(E Q D 2_{\alpha \beta 10}=30 \mathrm{~Gy} /\right.$ EQD $2_{\alpha \beta 3}=42 \mathrm{~Gy}$ ). Total dose and dose per fraction could also vary according to the clinical response observed at the time of HDRB.

Dose volume adaptation was manually achieved using graphical optimization (OncentraBrachy, Elekta Company, Elekta AB, Stockholm, Sweden) by dwell location and time variation (Figure 2). Patient stayed in supine position during all the hospitalization in a non-shielded room receiving specific care such as massages and antithrombotic therapy. The patient was transferred to her bed to the brachytherapy bunker for each fraction. After the last brachytherapy session and analgesic pre-medication, applicator and needles were removed, paying attention to the risk of vaginal and perineal bleeding. The patient left the hospital the day after in the absence of early complications.

\section{Follow-up and evaluation}

Patients were followed-up at first and 6 months after HDRB completion; then every 6 months alternatively by the radiation oncologist and the gynecologic surgeon. Surveillance consisted of clinical examination while CT, MRI, and PET were used in case of suspicion of local, regional, or distant progression. RCT and HDRB were proposed as definitive treatment, while post-RCT hysterectomy was proposed only in case of persistent residual disease or local recurrence.
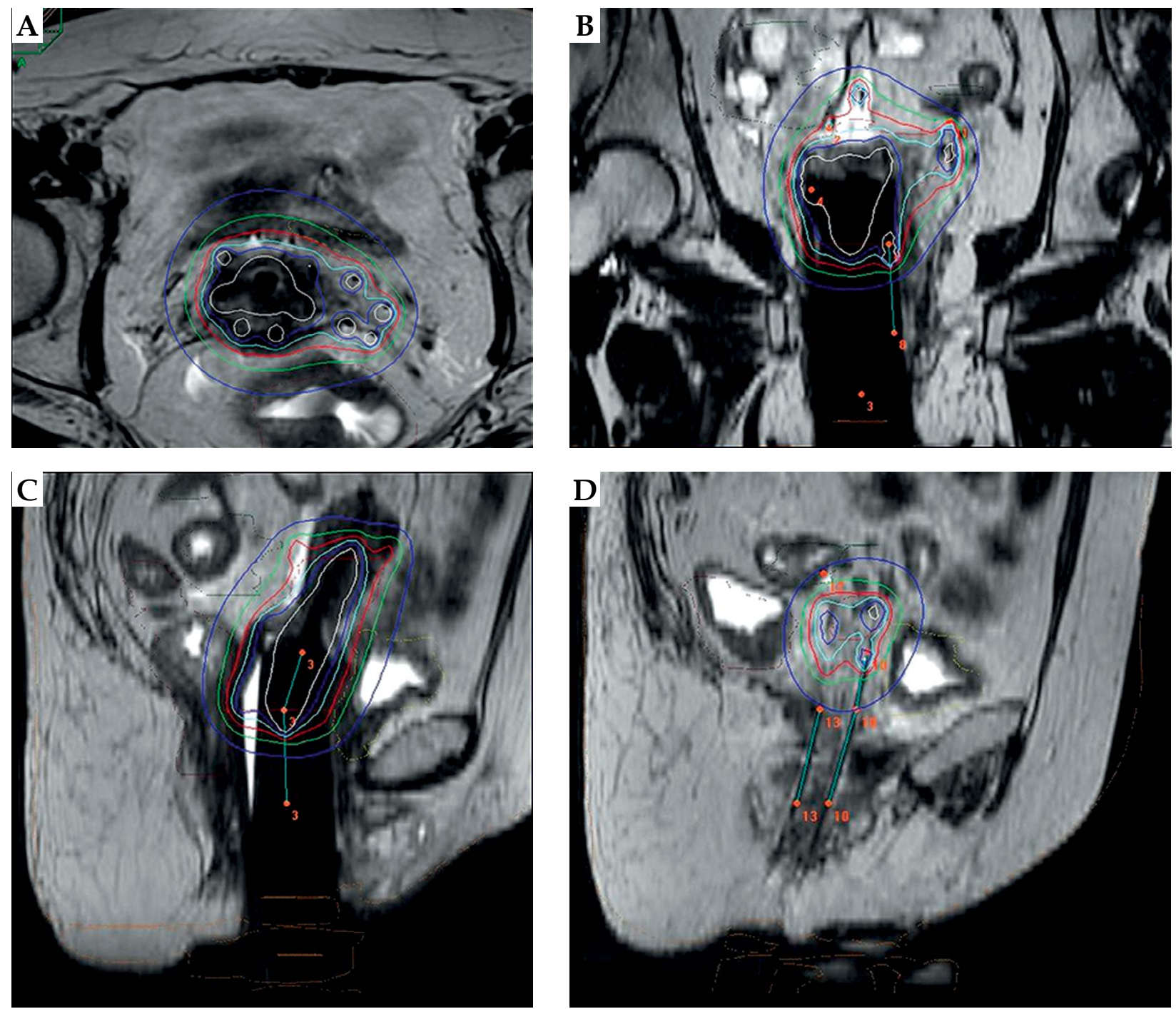

Fig. 2. Post-operative MRI-planning dose distribution analysis: A) transversal view, B) frontal view, C) mid-sagittal view,

D) para-sagittal view 
Acute toxicities occurring within the 6 months after HDRB were graded using the NCI-Common Toxicity Criteria version 4.0 (CTCV4.0) [12] based on the following items: urinary disorders (hematuria, increase in frequency, urgency, dysuria, nocturia, incontinence), gastro-intestinal disorders (diarrhea, proctitis), infections (urinary tract, kidney, uterine, and pelvic infections), hemorrhages (intra and post-operative, vaginal, retroperitoneal).

\section{Statistical analysis}

Quantitative data were represented as median, extremes, and standard deviation. Qualitative data were represented as percentage and extremes. For a subgroup of patients who had a median follow-up of 24 months, local-relapse free survival (LRFS), regional-relapse free survival (RRFS), and overall survival (OS) were calculated (Kaplan-Meier method - 24 and 36 months) with standard errors. The follow-up was calculated between the date of the first fraction of RCT, and the date of local recurrence

Table 1. Patient features

\begin{tabular}{|c|c|c|}
\hline Data & N/Median & $\% /[$ Min-max $]$ \\
\hline Age (years) & 56.4 & {$[27-79]$} \\
\hline \multicolumn{3}{|l|}{ Tumor stage } \\
\hline $\mathrm{T} 2 \mathrm{~b}$ & 23 & 69.7 \\
\hline T3a & 1 & 3.0 \\
\hline $\mathrm{T} 3 \mathrm{~b}$ & 6 & 18.2 \\
\hline $\mathrm{T} 4 \mathrm{a}$ & 3 & 9.1 \\
\hline \multicolumn{3}{|l|}{ Parametrial involvement } \\
\hline Right & 10 & 30.3 \\
\hline Left & 18 & 54.5 \\
\hline Bilateral & 5 & 15.2 \\
\hline Tumor size (mm) & 45 & [13-83] \\
\hline \multicolumn{3}{|l|}{ Lymph node status } \\
\hline $\mathrm{N}-$ & 18 & 54.5 \\
\hline $\mathrm{N}+$ & 15 & 45.5 \\
\hline \multicolumn{3}{|l|}{ Metastatic status } \\
\hline $\mathrm{M}-$ & 32 & 97.0 \\
\hline $\mathrm{M+}$ & 1 & 3.0 \\
\hline \multicolumn{3}{|l|}{ Histological type } \\
\hline Epidermoid carcinoma & 29 & 87.9 \\
\hline Well differentiated & 7 & 12.3 \\
\hline Moderately differentiated & 9 & 15.8 \\
\hline Poorly differentiated & 11 & 19.3 \\
\hline Undifferentiated & 1 & 1.8 \\
\hline Unknown & 1 & 1.8 \\
\hline Adenocarcinoma & 3 & 5.3 \\
\hline Other & 1 & 1.8 \\
\hline
\end{tabular}

and the date of last follow-up. Patients were censored at the moment of their death or their last follow-up.

\section{Results}

\section{Patients and treatments}

Patient and treatment characteristics are reported in Tables 1 and 2, respectively. Median age was 56.4 years (27-79). Histological type was squamous cell carcinoma, adenocarcinoma, and adenoid basal carcinoma for 29 pts $(87.9 \%), 3$ pts $(9.1 \%)$, and 1 pt $(3 \%)$, respectively. According to the UICC-TNM classification [10], patients were

Table 2. Treatment features

\begin{tabular}{|c|c|c|}
\hline Data & N/Median & $\% /[$ Min-max $]$ \\
\hline EBRT & 33 & 100 \\
\hline Dose per fraction (Gy) & 2 & {$[1.8-2.2]$} \\
\hline Total dose & 46 & {$[45.5-50.6]$} \\
\hline Concomitant CT & 32 & 97.0 \\
\hline Weekly cisplatin & 27 & 81.8 \\
\hline Weekly cisplatin-gemcitabine & 1 & 3.0 \\
\hline W1-W5 5FU-cisplatin & 1 & 3.0 \\
\hline Weekly carboplatin & 3 & 9.1 \\
\hline HDRB & 33 & 100 \\
\hline Number of parametrial needles & 4 & {$[2-6]$} \\
\hline 2 & 6 & 18.2 \\
\hline 3 & 2 & 6.1 \\
\hline 4 & 23 & 69.7 \\
\hline 6 & 2 & 6.1 \\
\hline Number of fractions & 3 & {$[3-5]$} \\
\hline 5 & 11 & 33.3 \\
\hline 3 & 22 & 66.7 \\
\hline Total dose & 21 & {$[21-27]$} \\
\hline 27 Gy & 1 & 3.0 \\
\hline 25 Gy & 9 & 27.3 \\
\hline $23 \mathrm{~Gy}$ & 1 & 3.0 \\
\hline 21 Gy & 22 & 66.7 \\
\hline $\begin{array}{l}\text { Interval time [EBRT - HDRB] } \\
\text { (days) }\end{array}$ & 17 & {$[5-47]$} \\
\hline Overall treatment time (days) & 54 & {$[44-72]$} \\
\hline Surgery & 3 & 9 \\
\hline $\begin{array}{l}\text { Time interval [HDRB - surgery] } \\
\text { (days) }\end{array}$ & 59 & {$[49-70]$} \\
\hline \multicolumn{3}{|l|}{ Pathologic response } \\
\hline No residual disease & 2 & 6 \\
\hline Macroscopic residual disease & 1 & 3 \\
\hline
\end{tabular}

5FU - 5-fluorouracile, EBRT-external beam radiation therapy, CT-chemotherapy, HDRB - high-dose-rate brachytherapy 
staged as: T2b (23 pts $-69.7 \%)$, T3a ( 1 pt $-3 \%)$, T3b (6 pts $-18.2 \%)$, and T4a (3 pts $-9.1 \%)$. Fifteen pts $(45.5 \%)$ presented pelvic-lymph node involvement diagnosed after MRI (6 pts) and/or PET-scan (9 pts). Median initial tumor size defined on MRI was $45 \mathrm{~mm}$ (13-83). Five patients had bilateral parametrial extension. Thirty-two patients (97\%) received concomitant chemotherapy: weekly cisplatin
(27 pts), weekly carboplatin (3 pts), weekly cisplatin-gemcitabine (1 pt), and 5 fluorouracil-cisplatin (W1-W5, $1 \mathrm{pt})$. Median EBRT dose was 46 Gy (45.5-50.6). Median HDRB dose was 21 Gy (21-27) in 3 to 5 fractions with a median treatment time of 2 days. Median number of parametrial implanted needles was 4 . Median overall treatment time was 54 days (44-72) and median delay between EBRT

Table 3. Dosimetric data reported for the 16 patients with CT-planification and the 17 patients with MRI-planification

\begin{tabular}{|c|c|c|c|c|c|c|c|}
\hline \multirow[t]{2}{*}{ Data } & & \multicolumn{3}{|c|}{ CT $(n=16)$} & \multicolumn{3}{|c|}{ MRI $(n=17)$} \\
\hline & & Median & Min-max & SD & Median & Min-max & SD \\
\hline CTV & $(\mathrm{cc})$ & 52.6 & $28.5-74.3$ & 13.1 & - & - & - \\
\hline \multirow[t]{2}{*}{ D90 ${ }_{\text {CTV }}$} & $(\%)$ & 118.0 & $108.0-127.6$ & 5 & - & - & - \\
\hline & (Gy) & 28.1 & $22.7-31.9$ & 3 & - & - & - \\
\hline D90 ${ }_{\text {CTV }} E Q D 2_{\alpha \beta 10}$ & (Gy) & 82.9 & $78.2-96.5$ & 5.7 & - & - & - \\
\hline HR-CTV & (cc) & - & - & - & 31.9 & $17.1-58.0$ & 11.7 \\
\hline \multirow[t]{2}{*}{ D90 ${ }_{\text {HR-CTV }}$} & $(\%)$ & - & - & - & 122.4 & $107.9-135.9$ & 7.2 \\
\hline & (Gy) & - & - & - & 25.7 & $22.7-28.5$ & 1.5 \\
\hline $\mathrm{EQD2} 2_{\alpha \beta 10} \mathrm{D} 90_{\mathrm{HR}-\mathrm{CTV}}$ & (Gy) & - & - & - & 84.8 & $80.6-91.4$ & 3.1 \\
\hline IR-CTV & $(\mathrm{cc})$ & - & - & - & 83.1 & $53.8-129$ & 19.6 \\
\hline \multirow[t]{2}{*}{ D90 IR-CTV } & $(\%)$ & - & - & - & 88.0 & $67.8-106.0$ & 10.9 \\
\hline & (Gy) & - & - & - & 18.3 & $12.6-22.3$ & 3 \\
\hline $\mathrm{EQD2}_{\alpha \beta 10} \mathrm{D} 90_{\mathrm{IR}-\mathrm{CTV}}$ & (Gy) & - & - & - & 70.5 & $59.9-78.3$ & 5.2 \\
\hline $\mathrm{V}_{100}$ & $(\%)$ & 99.0 & $95.3-100$ & 1.2 & 98.6 & $92.3-99.3$ & 2.2 \\
\hline$V_{150}$ & (\%) & 52.2 & $28.3-69.4$ & 11.2 & 57.2 & $48.5-67.1$ & 5.3 \\
\hline$V_{200}$ & $(\%)$ & 16.1 & $11.6-39.6$ & 7.5 & 23.9 & $20.5-34.0$ & 3.6 \\
\hline \multicolumn{8}{|l|}{ Bladder } \\
\hline $\mathrm{D} 0.1 \mathrm{cc} b$ & $(\%)$ & 100.8 & $84.8-125.1$ & 10.2 & 92.8 & $82.3-103.7$ & 6 \\
\hline $\mathrm{D} 1 \mathrm{cc} c_{\mathrm{b}}$ & (\%) & 88.6 & $72.0-106.5$ & 8.8 & 85.0 & $76.7-90.5$ & 4.5 \\
\hline $\mathrm{D} 2 \mathrm{cc}_{\mathrm{b}}$ & $(\%)$ & 83.8 & $65.0-100.4$ & 9.5 & 79.6 & $71.6-85.0$ & 4.5 \\
\hline $\mathrm{EQD} 2_{\alpha \beta 3} \mathrm{D} 2 \mathrm{cc} c_{\mathrm{b}}$ & (Gy) & 76.8 & $66.6-90.9$ & 6 & 74.5 & 70.3-78.0 & 2.3 \\
\hline \multicolumn{8}{|l|}{ Rectum } \\
\hline D0.1cc & $(\%)$ & 86.5 & $29.6-104.0$ & 21.1 & 82.2 & $52.1-90.7$ & 11 \\
\hline $\mathrm{D} 1 \mathrm{cc} \mathrm{r}_{\mathrm{r}}$ & (\%) & 72.6 & $21.3-85.3$ & 18.4 & 68.4 & $42.3-79.1$ & 10.9 \\
\hline $\mathrm{D} 2 \mathrm{cc}_{\mathrm{r}}$ & $(\%)$ & 65.1 & $19.0-77.8$ & 15.1 & 60.7 & $38.1-75.1$ & 10.5 \\
\hline $\mathrm{EQD2} 2_{\alpha \beta 3} \mathrm{D} 2 \mathrm{cc} \mathrm{r}_{\mathrm{r}}$ & (Gy) & 66.4 & $51.9-77.4$ & 7.6 & 64.3 & $55.1-72.0$ & 4.6 \\
\hline \multicolumn{8}{|l|}{ Sigmoid } \\
\hline $\mathrm{D} 0.1 \mathrm{cc}_{\mathrm{s}}$ & $(\%)$ & 78.8 & $61.1-129.6$ & 23.9 & 75.2 & $35.1-93.8$ & 17.9 \\
\hline $\mathrm{D} 1 \mathrm{cc} \mathrm{s}_{\mathrm{s}}$ & (\%) & 70.0 & $50.2-106.0$ & 17.6 & 56.5 & $29.2-81.5$ & 15.6 \\
\hline $\mathrm{D} 2 \mathrm{Cc}_{\mathrm{s}}$ & (\%) & 60.9 & $46.0-97.8$ & 17.2 & 52.4 & $24.5-73.7$ & 14.4 \\
\hline $\mathrm{EQD} 2_{\alpha \beta 3} \mathrm{D} 2 \mathrm{cc}_{\mathrm{s}}$ & (Gy) & 59.8 & $53.4-81.1$ & 8.5 & 60.7 & $50.9-70.3$ & 5.7 \\
\hline
\end{tabular}

$C T$ - planification based on CT-scan $=16$ patients (pts): MRI- planification based on MRI $=17$ pts: $C T V$ - clinical target volume: HR-CTV - high-risk CTV: IR-CTV intermediate-risk CTV; D90 $C T V$ - dose delivered to $90 \%$ of the CTV; D90 ${ }_{H R-C T V}$ - dose delivered to $90 \%$ of the HR-CTV; D90 IR-CTV - dose delivered to $90 \%$ of the IR-CTV; $E Q D 2_{\alpha \beta 30}$ - equivalent dose at $2 \mathrm{~Gy}$ per fraction for $\alpha \beta=10$ (tumor); EQD2 $2_{\alpha \beta 3}$ - equivalent dose at $2 \mathrm{~Gy}$ per fraction for $\alpha \beta=3$ (normal tissues); $V_{100}$ - volume receiving $100 \%$ of the prescribed dose; $V_{150}$ - volume receiving $150 \%$ of the prescribed dose; $V_{200}$ - volume receiving $200 \%$ of the prescribed dose; D0.1cc - dose delivered to $0.1 \%$ of the bladder volume; $D 0.1 c c_{r}$ - dose delivered to $0.1 \%$ of the rectum volume; $D 0.1 c c_{s}$ - dose delivered to $0.1 \%$ of the sigmoid volume; DIcc - - dose delivered

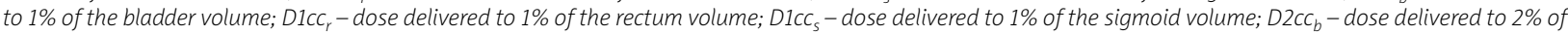
the bladder volume; D2Cc - dose delivered to $2 \%$ of the rectum volume; D2ccs - dose delivered to $2 \%$ of the sigmoid volume; SD - standard deviation 
Table 4. Acute toxicities occurring within the 6 months after HDRB using the CTCAE 4.0.; results expressed as a number of events and a percentage of patients. Total number of toxicities expressed as a number of patients in whom at least one toxicity scored G0, G1, G2, G3, or G4 occurred

\begin{tabular}{|c|c|c|c|c|c|c|c|c|c|c|}
\hline \multirow[t]{2}{*}{ Toxicities } & \multicolumn{2}{|c|}{ G0 } & \multicolumn{2}{|c|}{ G1 } & \multicolumn{2}{|c|}{ G2 } & \multicolumn{2}{|c|}{ G3 } & \multicolumn{2}{|c|}{ G4 } \\
\hline & pts & $\%$ & pts & $\%$ & pts & $\%$ & pts & $\%$ & pts & $\%$ \\
\hline GU & 25 & 75.8 & 8 & 24.2 & 0 & 0 & 0 & 0 & 0 & 0 \\
\hline $\mathrm{Gl}$ & 24 & 72.7 & 9 & 27.3 & 0 & 0 & 0 & 0 & 0 & 0 \\
\hline Bleeding & 32 & 97.0 & 0 & 0 & 0 & 0 & 1 & 3 & 0 & 0 \\
\hline Infectious & 29 & 87.9 & 0 & 0 & 2 & 6.1 & 2 & 6.1 & 0 & 0 \\
\hline Total & 16 & 48.5 & 14 & 42.4 & 2 & 6.1 & 3 & 9.1 & 0 & 0 \\
\hline
\end{tabular}

GU - genito-urinary toxicities, GI - gastro-intestinal toxicities

and HDRB was 17 days (5-47). Three patients underwent adjuvant hysterectomy after a median time interval [HDRB-surgery] of 59 days (49-70): 2 pts presented a complete pathological response and macroscopic residual disease was observed in 1 pt. Median follow-up for the whole cohort was 14 months (6-51) while 24 pts $(72.7 \%)$ had a median follow-up of 24 months (8-51).

\section{Dosimetric data}

Dosimetric data are reported in Table 3. Planification was performed on CT for 16 pts $(48.5 \%)$ and on MRI for 17 pts $(51.5 \%)$. Median CTV, HR-CTV ${ }_{\text {MRI }}$, and IR-CTV were 52.6 cc (28.5-74.3), 31.9 cc (17.1-58.0), and $83.1 \mathrm{cc}$ (53.8-129.0), respectively. EQD2 ${ }_{\alpha \beta 10}$ for $\mathrm{D} 90_{\mathrm{CTV}}, \mathrm{D} 90_{\mathrm{HR}-\mathrm{CTV}}$ and $\mathrm{D} 90_{\mathrm{IR}-\mathrm{CTV}}$ were $82.9 \mathrm{~Gy}$ (78.2-96.5), 84.8 Gy (80.6-91.4) and 70.5 Gy (59.9-78.3), respectively. Median V100\% and median V100\% ${ }_{\text {MRI }}$ were $99.0 \%$ (95.3-100.0) and 98.6\% (92.3-99.3), respectively.

For OARs, D2cc EQD2 ${ }_{\alpha \beta 3}$ of the whole cohort for bladder, rectum, and sigmoid were 75.5 Gy (66.6-90.9), 64.4 Gy (51.9-77.4), and 60.4 Gy (50.9-81.1), respectively. D2cc EQD2 ${ }_{\alpha \beta 3}$ of CT-planning patients for bladder, rectum, and sigmoid were 76.8 Gy (66.6-90.9), 66.4 Gy (51.9-77.4), and 59.8 Gy (53.4-81.1), respectively. D2cc EQD2 ${ }_{\alpha \beta 3}$ of MRI-planning patients for bladder, rectum, and sigmoid were 74.5 Gy (70.3-78.0), 64.3 Gy (55.1-72.0), and 60.7 Gy (50.9-70.3), respectively.

\section{Acute toxicities}

Acute toxicities occurring within the 6 months after HDRB are reported in Table 4. Urinary and gastro-intestinal disorders occurred in 8 pts $(100 \%$ G1) and 9 pts (100\% G1), respectively. Infections were scored G2 for 2 pts (urinary) and G3 for 2 pts (urinary and endometritis). One patient presented a vaginal bleeding at the time of applicator withdrawal (G3 because of blood transfusion) but no bleeding was observed due to parametrial implant.

\section{Survival analysis}

Among 24 pts who had a median follow-up of 24 months, the staging was: T2b (17 pts), T3b (6 pts), and T4a (1 pt). For this sub-group, 2-year LRFS rate was $86.8 \%$ $( \pm 14.2)$ remaining stable at 3 years. Two-year RRFS and
OS rates were $88.8 \%( \pm 15.4)$ and $94.1 \%( \pm 20.4)$, respectively; while at 3 years, those survival rates were 76.1 $( \pm 27)$ and $85.6( \pm 19.4)$, respectively.

\section{Discussion}

Currently, standard brachytherapy technique is based on pulsed dose rate (PDR) or HDR [13]. In case of parametrial involvement, the combination between endocavitary and interstitial brachytherapy is generating a lot of interest [14]. Indeed, increasing the "field" number makes dose distribution optimization easier and more accurate. However, the parametrial boost technique remains under discussion. Historically, parametrial boost was performed through EBRT, before or after brachytherapy increasing not only treatment protraction but also the risk of overlap of irradiated volumes between EBRT-boost and cervical brachytherapy. Besides those clinical considerations, Mohamed et al. compared parametrial boost dose distribution delivered either by EBRT or interstitial brachytherapy (ISBT) [9]. The authors confirmed that ISBT was superior to EBRT in terms of organ sparing (less normal tissue exposure to intermediate doses $-\mathrm{V}_{60}$ ) and target coverage (more conformal).

Currently, interstitial parametrial brachytherapy boost can be performed through a transvaginal or a transperineal technique. In this study, the results of a transperineal approach were reported.

Regarding dose distribution analysis, $\mathrm{D} 2 \mathrm{cc} \mathrm{EQD} 2_{\alpha \beta 3}$ of the bladder matched the GEC-ESTRO dose constrain recommendations (<90 Gy), constraints were also respected for D2cc EQD2 ${ }_{\alpha \beta 3}$ of the rectum and sigmoid (< $75 \mathrm{~Gy}$ ) [15]. However, considering more rigorous OAR dose constraints as discussed during the $5^{\text {th }}$ EMBRACE Annual Meeting in Vienna $\left(17^{\text {th }}-18^{\text {th }}\right.$ January, 2014), bladder D2cc EQD2 ${ }_{\alpha \beta 3}$ was superior to 80 Gy for 3 patients $(9 \%)$, rectum D2cc EQD2 $2_{\alpha \beta 3}$ was superior to 70 Gy for 6 pts $(18 \%)$, and sigmoid D2cc EQD2 $2_{\alpha \beta 3}$ was superior to 75 Gy for $1 \mathrm{pt}(3 \%)$.

Beside the transperineal approach described in this study, mainly Vienna and Aarhus teams presented a transvaginal technique. Berger et al. reported dosimetric and clinical outcome of 6 patients who underwent oblique needle implantation from the vagina using a modified Vienna applicator [16]. HR-CTVs of mean 50 cc were treated with mean D90 of $86 \mathrm{~Gy}$. Based on 2 to 3 implants of 
PDR brachytherapy Lindegaard et al. described an oblique needle implantation from the vagina [14]. The authors obtained a mean $\mathrm{D} 90_{\mathrm{HR}-\mathrm{CTV}}$ of $87 \mathrm{~Gy}$ in tumors with a mean HR-CTV of 53 cc. More recently, Mohamed et al. reported the dosimetric results of a cohort of 23 patients who underwent 2 PDR implants (1 week apart) based on endocavitary and oblique needle implantation from the vagina [9]. The authors calculated D90 $0_{\text {HR-CTV }}>84$ Gy for all patients.

Transperineal interstitial approach has been criticized for bleeding risk due to vascular injury at the time of needle insertion. In the present study, one patient presented vaginal bleeding at the time of applicator withdrawal but no bleeding was observed due to parametrial implant. Indeed, at least six experimented brachytherapy teams analyzed the feasibility of transperineal brachytherapy technique for gynecological malignancies [17,18,19]. Among a total of 481 patients, no severe bleeding complications were reported. Bleeding risk remains a rare but potential event, which is strongly correlated to inter-individual heterogeneity in blood vessel architecture.

In this study, we reported the results of a single implant allowing delivering 3 to 5 fractions based on HDRB. According to our knowledge, this is the first analysis of such approach while combination between endocavitary and interstitial brachytherapy was mainly reported using PDR brachytherapy and always with at least 2 implants 1 week apart. Utrecht and Aarhus teams described their results. Jürgenliemk-Schulz et al. reported the results of 24 patients with a mean $\mathrm{D} 90_{\mathrm{HR}-\mathrm{CTV}}$ of 84 Gy [20]. Nomden et al. analyzed the dosimetric results of 20 patients treated with two pulsed dose rate applications combining endocavitary and interstitial, and noticed a mean D90 HR-CTV of 83.9 Gy [7]. More recently, Aarhus University published the results of 71 patients treated with 2 PDR implants with a mean D90 $0_{\text {HR-CTV }}$ of 94.5 Gy while mean EQD2 D2cc of bladder, rectum, and sigmoid were 68.5, 61.0, and 64.9 Gy, respectively [21]. Fokdal et al. reported only minor morbidity, which was resolved at a 3 month follow- up after 3 implants of endocavitary and interstitial PDR BT in 58 patients [22].

The American Brachytherapy Society recommends a cumulative delivered dose of approximately 80-90 Gy as a component of the definitive treatment of locally advanced cervix carcinoma while precise applicator placement remains necessary to maximize the probability of achieving local control without major side effects [23]. For HDRB, the recommended D90 EQD2 ${ }_{\alpha \beta 10}$ is 80 to $90 \mathrm{~Gy}$, depending on tumor size at the time of brachytherapy $[24,25]$. Dimopoulos et al. demonstrated that a local control superior to $90 \%$ can be achieved if the D90 EQD2 ${ }_{\alpha \beta 10}$ was at least 86 Gy [26]. Pötter et al. study's generated satisfying results in terms of local control after EBRT with or without chemotherapy followed by HDRB $(4 \times 7 \mathrm{~Gy})$ with the objective of D90 ${ }_{\mathrm{HR}-\mathrm{CTV}} \mathrm{EQD} 2_{\alpha \beta 10}>85 \mathrm{~Gy}$. In this study, brachytherapy was guided by clinical examination and MRI and the authors reported a 3-year local-control rate of $96 \%$ and $86 \%$ for stage IIB and IIIB, respectively [27]. In our study, median $\mathrm{D} 90_{\mathrm{CTV}} \mathrm{EQD} 2_{\alpha \beta 10}$ (CT-scan data) and median D90 ${ }_{\mathrm{HR}-\mathrm{CTV}} \mathrm{EQD} 2_{\alpha \beta 10}$ (MRI data) ranged between 80 to $90 \mathrm{~Gy}$ (82.5 Gy and $84.8 \mathrm{~Gy}$, respectively) in respect to the GEC-ESTRO dose constraint recommen- dations [15]. No patient received less than $80 \mathrm{~Gy}$ and we calculated a D90 $0_{\mathrm{HR}-\mathrm{CTV}} \mathrm{EQD} 2_{\alpha \beta 10}>84 \mathrm{~Gy}$ for 12 patients $(71 \%)$ in the MRI-planning group. However, Tanderup et al. recently suggested that $\mathrm{D} 90_{\mathrm{HR}-\mathrm{CTV}} \mathrm{EQD} 2_{\alpha \beta 10}>90 \mathrm{~Gy}$ and $>85$ Gy for small and large residual tumors, respectively, could improve local control rate by 3-4\% [28]. In order to reach the dose levels suggested by Tanderup et al., it would be necessary to increase our prescribed dose up to $24 \mathrm{~Gy}$ in 3 fractions over 2 days. Computer simulation modeling revealed that this increase would allow reaching a $\mathrm{D} 90_{\mathrm{HR}}$ CTV EQD2 ${ }_{\alpha \beta 10}$ of 94.5 Gy (86.2-102.7) with an acceptable increase of D2cc EQD2 ${ }_{\alpha \beta 3}$ for OARs (data not shown).

However, those dose constraints derived from different prescription dose protocols, which do not take into account treatment protraction, which could significantly impact on the final biological effect. Here, we propose a single implant of HDRB for cervical and parametrial disease with 3 fractions over 2 consecutive days. Biological impact on target volume and OARs of 21 Gy delivered over 2 days $(3 \times 7 \mathrm{~Gy})$ could be different than the same physical dose delivered during a longer treatment scheme ( 2 to 3 weeks) and 3 different implants.

\section{Conclusions}

Short follow-up, small cohort of patient, and retrospective approach are the principal limiting factors of this study. However, concomitant cervical and transperineal parametrial HDRB boost for locally advanced cervical cancer appears feasible and safe with no specific acute toxicity compare to cervix HDRB alone. More specifically, transperineal parametrial HDRB boost does not increase the risk of bleeding. This technique allows respecting dose constraints for HR-CTV and OARs. Longer follow-up and larger patient cohort will be necessary to confirm this approach.

\section{Disclosure}

Prof. Jean-Michel Hannoun-Levi declares association with ELEKTA, related to the patent No 8834339: Assembly for performing brachytherapy treatment of a tumour tissue in an animal body (http://www.google.com.au/patents/ US8834339 [1]).

The other authors report no conflict of interest.

\section{References}

1. Ferlay J, Soerjomataram I, Dikshit R et al. Cancer incidence and mortality worldwide: Sources, methods and major patterns in GLOBOCAN 2012. Int J Cancer 2015; 136: E359-386.

2. Lanciano RM, Martz K, Coia LR et al. Tumor and treatment factors improving outcome in stage III-B cervix cancer. Int J Radiat Oncol Biol Phys 1991; 20: 95-100.

3. Han K, Milosevic M, Fyles A et al. Trends in the utilization of brachytherapy in cervical cancer in the United States. Int J Radiat Oncol Biol Phys 2013; 87: 111-119.

4. Gill BS, Lin JF, Krivak TC et al. National Cancer Data Base analysis of radiation therapy consolidation modality for cervical cancer: The impact of new technological advancements. Int J Radiat Oncol Biol Phys 2014; 90: 1083-1090.

5. Dimopoulos JC, Kirisits C, Petric P et al. The Vienna applicator for combined intracavitary and interstitial brachytherapy 
of cervical cancer: clinical feasibility and preliminary results. Int J Radiat Oncol Biol Phys 2006; 66: 83-90.

6. Kirisits C, Lang S, Dimopoulos J et al. The Vienna applicator for combined intracavitary and interstitial brachytherapy of cervical cancer: design, application, treatment planning, and dosimetric results. Int J Radiat Oncol Biol Phys 2006; 65: 624-630.

7. Nomden CN, de Leeuw AA, Moerland MA et al. Clinical use of the Utrecht applicator for combined intracavitary/interstitial brachytherapy treatment in locally advanced cervical cancer. Int J Radiat Oncol Biol Phys 2012; 82: 1424-1430.

8. Hannoun-Levi J-M, Chand-Fouche M-E, Gautier M et al. Interstitial preoperative high-dose-rate brachytherapy for early stage cervical cancer: dose-volume histogram parameters, pathologic response and early clinical outcome. Brachytherapy 2013; 12: 48-155.

9. Mohamed S, Kallehauge J, Fokdal L et al. Parametrial boosting in locally advanced cervical cancer: Combined intracavitary/interstitial brachytherapy vs. intracavitary brachytherapy plus external beam radiotherapy. Brachytherapy 2015; 14: 23-28.

10. Sobin L, Gospodarowicz M, Wittekind CE. TNM Classification of Malignant Tumors. $7^{\text {th }}$ ed. Wiley-Blackwell, Oxford 2009.

11. Haie-Meder C, Pötter R, Van Limbergen E et al. Recommendations from Gynaecological (GYN) GEC-ESTRO Working Group (I): concepts and terms in 3D image based 3D treatment planning in cervix cancer brachytherapy with emphasis on MRI assessment of GTV and CTV. Radiother Oncol 2005; 74: $235-245$.

12. CTCAE.4. National Cancer Institute Common Terminology Criteria for Adverse Events v4.0 NCI, NIH, DHHS. May 29, 2009. NIH publication \# 09-7473.

13. Miglierini P, Malhaire J-P, Goasduff $G$ et al. Cervix cancer brachytherapy: high dose rate. Cancer Radiother 2014; 18: 452-457.

14. Lindegaard JC, Tanderup K. Counterpoint: Time to retire the parametrial boost. Brachytherapy 2012; 11: 80-83.

15. Pötter R, Haie-Meder C, Van Limbergen E et al. Recommendations from gynaecological (GYN) GEC ESTRO working group (II): concepts and terms in 3D image-based treatment planning in cervix cancer brachytherapy - 3D dose volume parameters and aspects of 3D image-based anatomy, radiation physics, radiobiology. Radiother Oncol 2006; 78: 67-77.

16. Berger D, Pötter R, Dimopoulos JCA et al. New Vienna applicator design for distal parametrial disease in cervical cancer Brachytherapy 2010; 9: S23-S102.

17. Martinez A, Cox RS, Edmundson GK. A multiple-site perineal applicator (MUPIT) for treatment of prostatic, anorectal, and gynecologic malignancies. Int J Radiat Oncol Biol Phys 1984; 10: 297-305.

18. Weitmann HD, Knocke TH, Waldhäusl C et al. Ultrasoundguided interstitial brachytherapy in the treatment of advanced vaginal recurrences from cervical and endometrial carcinoma. Strahlenther Onkol 2006; 182: 86-95.

19. Mahantshetty U, Shrivastava S, Kalyani N et al. Templatebased high-dose-rate interstitial brachytherapy in gynecologic cancers: a single institutional experience. Brachytherapy 2014; 13 : 337-342

20. Jürgenliemk-Schulz IM, Tersteeg RJ, Roesink JM et al. MRI-guided treatment-planning optimization in intracavitary or combined intracavitary/interstitial PDR brachytherapy using tandem ovoid applicators in locally advanced cervical cancer. Radiother Oncol 2009; 93: 322-330.

21. Mohamed S, Nielsen SK, Fokdal LU et al. Feasibility of applying a single treatment plan for both fractions in PDR image guided brachytherapy in cervix cancer. Radiother Oncol 2013; 107: 32-38

22. Fokdal L, Tanderup K, Hokland SB et al. Clinical feasibility of combined intracavitary/interstitial brachytherapy in locally advanced cervical cancer employing MRI with a tan$\mathrm{dem} / \mathrm{ring}$ applicator in situ and virtual preplanning of the interstitial component. Radiother Oncol 2013; 107: 63-68.

23. Viswanathan AN, Thomadsen B. American Brachytherapy Society Cervical Cancer Recommendations Committee, American Brachytherapy Society. American Brachytherapy Society consensus guidelines for locally advanced carcinoma of the cervix. Part I: general principles. Brachytherapy 2012; 11: 33-46.

24. Viswanathan AN, Beriwal S, De Los Santos JF et al. American Brachytherapy Society consensus guidelines for locally advanced carcinoma of the cervix. Part II: high-dose-rate brachytherapy. Brachytherapy 2012; 11: 47-52.

25. Lee LJ, Das IJ, Higgins SA et al. American Brachytherapy Society consensus guidelines for locally advanced carcinoma of the cervix. Part III: low-dose-rate and pulsed-dose-rate brachytherapy. Brachytherapy 2012; 11: 53-57.

26. Dimopoulos JC, Pötter R, Lang $S$ et al. Dose-effect relationship for local control of cervical cancer by magnetic resonance image-guided brachytherapy. Radiother Oncol 2009; 93: 311-315.

27. Pötter R, Georg P, Dimopoulos JC et al. Clinical outcome of protocol based image (MRI) guided adaptive brachytherapy combined with 3D conformal radiotherapy with or without chemotherapy in patients with locally advanced cervical cancer. Radiother Oncol 2011; 100: 116-123.

28. Tanderup K, Fokdal L, Sturdza A. Dose and Volume Response for Local Control in Locally Advanced Cervical Cancer Treated With EBRT Combined With MRI-Guided Adaptive Brachytherapy. Int J Radiat Oncol Biol Phys 2014 Suppl. Abstract S92. 